\title{
FORMACIÓN ÉTICA COMO SOPORTE DEL DESARROLLO MORAL DEL ALUMNO DE CARRERAS DE CIENCIAS DE LA SALUD: HACIA UNA ENSEÑANZA CENTRADA EN LAS NECESIDADES DEL ESTUDIANTE COMO PERSONA
}

\begin{abstract}
Alberto Perales ${ }^{1, a}$
\section{RESUMEN}

Tomando como base las encuestas epidemiológicas sobre salud mental aplicadas a estudiantes de las Ciencias de la Salud de la Facultad de Medicina (Medicina, Enfermería, Obstetricia, Nutrición y Tecnología Médica) de la Universidad Nacional Mayor de San Marcos, el autor postula que existen dos formas de entender las necesidades de enseñanza de la ética: 1) Como estrategia curricular, con información teórica a través de cursos específicos; y, 2) Como soporte al desarrollo moral de la personalidad con diversas metódicas innovadoras. Apoyándose en los resultados de las encuestas citadas, que evidencian prevalencias de vida de intento suicida y conductas disociales mayores que en población general, se plantea que tal enseñanza debe planificarse para dos grupos de alumnos con diferentes necesidades: 1) Aquellos que sin patología de salud mental pueden beneficiarse con la estrategia curricular regular; y, 2) Aquellos que, presentando problemas de salud mental e indicadores de dificultades en su conducta social, deben, además, ser ayudados con técnicas orientadas a ofrecerles apoyo en su desarrollo moral. Y que este reto educativo debe ser responsabilidad de la universidad del siglo XXI, bajo la pregunta de ¿qué necesita nuestro país, profesionales de la salud que sepan ética o que se comporten éticamente?
\end{abstract}

Palabras clave: Educación; Ética; Salud Mental; Estudiantes del Área de la Salud; Perú (fuente: DeCS BIREME).

\section{ETHICAL TRAINING AS A SUPPORT FOR THE MORAL DEVELOPMENT OF STUDENTS IN HEALTH SCIENCES PROGRAMS: TOWARDS A TEACHING FOCUSED ON THE NEEDS OF THE STUDENT AS A PERSON}

\begin{abstract}
Based on the epidemiological surveys on mental health applied to Health Science students of the School of Medicine (Medicine, Nursing, Obstetrics, Nutrition, and Medical Technology) of Universidad Nacional Mayor de San Marcos, the author postulates that there are two ways to understand the teaching needs of ethics: 1) as a curricular strategy, with theoretical information through specific courses; and, 2) as a support to the moral development of the personality with diverse innovative methods. Based on the results of the surveys mentioned, which show lifetime prevalence of suicide attempts and dissocial behaviors greater than in the general population, it is proposed that such teaching shall be planned for two groups of students with different needs: 1) those without a mental health pathology and who can benefit from the regular curricular strategy; and, 2) those who, with mental health problems and indicators of difficulties in their social behavior, shall also be helped with techniques aimed at offering them support in their moral development. And that this educational challenge must be the responsibility of the university of the 21st century, under the question: What does our country need, health professionals who know ethics or who behave ethically?
\end{abstract}

Keywords: Education; Ethics; Mental Health; health-Area Students; Peru (source: MeSH NLM).

\section{INTRODUCCIÓN}

Hablar de ética en educación médica y ciencias de la salud es hablar de actitudes y comportamientos morales que se espera el estudiante adquiera durante sus estudios de pregrado para, ulteriormente, cumplir adecuadamente su rol profesional. Clásicamente, la universidad ha intentado lograr este propósito implementando técnicas variadas de enseñanza de la ética y materias vinculadas ${ }^{(1-3)}$. La importancia y necesidad de tal quehacer no se discute pero se debate la eficacia de los procedimientos empleados ${ }^{(4,5)}$.

Por mi parte, deseo desde un comienzo precisar claramente mi posición al respecto. Considero que existen dos formas de entender las necesidades de enseñanza de la ética. Primero, como estrategia curricular, con información teórica a través

\footnotetext{
Instituto de Ética en Salud, Facultad de Medicina, Universidad Nacional Mayor de San Marcos. Lima, Perú.

a Médico psiquiatra, doctor en Medicina.

Recibido: 21/02/2019 Aprobado: 06/03/2019 En línea: 21/03/2019
}

Citar como: Perales A. Formación ética como soporte del desarrollo moral del alumno de carreras de Ciencias de la Salud: Hacia una enseñanza centrada en las necesidades del estudiante como persona. Rev Peru Med Exp Salud Publica. 2019;36(1):100-5. doi:10.17843/rpmesp.2019.361.4314. 
de cursos específicos sobre Filosofía de la Medicina, Ética, Deontología, Bioética, y otros, por medio de seminarios, talleres, foros, discusión de casos. Y segundo, como soporte al desarrollo moral de la personalidad con diversas metódicas innovadoras.

Un esfuerzo serio para mejorar la calidad de tal proceso educativo debe ser parte de una fundamentada teoría del desarrollo moral del hombre, pues no todos los estudiantes tienen la suerte de ingresar a la universidad en condiciones morales óptimas. Más aún, muchos lo hacen con diversos problemas de salud mental que incidirán negativamente en su futuro desempeño académico y en la observancia de una ética profesional.

\section{RETOS Y PERSPECTIVAS ÉTICAS DE LA MEDICINA Y LAS CIENCIAS DE LA SALUD}

Analizar el problema de la enseñanza de la ética en la medicina y las ciencias de la salud y plantear estrategias para su afronte es una tarea urgente y compleja. Intentarlo es apuntar al problema medular de nuestra profesión. Tal es el reto que se ha impuesto la Revista Peruana de Medicina Experimental y Salud Pública, al organizar el presente simposio.

En la Grecia antigua el concepto de telos señalaba que en la vida todo tiene un propósito o finalidad última. Aristóteles utiliza esta noción en la física y luego en la biología y señala que «nosotros (los hombres) somos, en algún sentido, un fin» (telos) ${ }^{(6)}$. De ello se deriva que para comprender realmente lo que «algo» es, debemos primero comprender su finalidad, su telos.

En el curso de la vida, el ser humano va configurando su conciencia moral e internalizando principios, valores y reglas que regulan el comportamiento durante todo su devenir, aunque en diferentes formas y grados: en su hogar, en la escuela, en la universidad, en su centro laboral, en su espacio social y por medio de normas o leyes que el Estado promulga para modelar la conducta ciudadana.

La pregunta que entonces debiéramos plantearnos es ¿por qué si el hombre conoce las buenas costumbres, los principios y valores morales para guiar su comportamiento hacia el bien, no siempre lo hace?

Mi particular punto de vista, desde que en $1988^{(7)}, 1989^{(8)}$ y $1993^{(9)}$, describí los tres problemas de salud mental más importantes en el Perú: 1) El subdesarrollo y la pobreza; 2) La corrupción generalizada; y, 3) La violencia; los cuales, en el fondo, son problemas éticos, es que toda sociedad constituye un sui-géneris experimento humano que, mediante un proceso histórico de ensayo-error, intenta perfeccionar sus métodos y costumbres de vida (ethos) para adaptarse a las demandas del entorno y evolucionar como grupo social hacia una sociedad justa y moralmente íntegra.

Sin embargo, según Ortiz, las diversas estrategias que las sociedades humanas han utilizado en tal propósito han fracasado ${ }^{(10)}$. La dificultad mayor en este proceso social evolutivo, deriva de la actuación de grupos humanos de poder circunstancial, que lejos de contribuir al desarrollo natural de la sociedad, introducen variables extrañas por intereses particulares de auto-beneficio que, ajenos a los objetivos del telos social, corrompen su evolución natural.

Por ello, la hipótesis que sustenta esta propuesta es que el bien y el mal no son entes abstractos sino problemas sociales que se expresan a través de acciones humanas. Por ende, no se podrá comprender ni moralizar (a un ser o grupo humano, cualquiera que fuere) sin tener en cuenta las conexiones con su entorno sociocultural e histórico en el que se halle.

Bacon, dijo en su momento: «Nuestro poder va tan lejos como nuestro saber». Saber es poder, investigar es saber; por tanto, investigar es poder. Y, como todo poder, puede corromperse y corromper, desviándose así de sus fines, que apuntan a perfeccionar lo más humano del ser humano. Por su parte, Frei Betto, el sacerdote y filósofo brasileño, planteó un razonamiento sugestivo al respecto: «Démosle a una persona una tajada de poder y sabremos quién es esa persona. El poder, al contrario de lo que se dice, no cambia a las personas: hace que se revelen. Así como el alcohol, el poder embriaga y, a veces, puede hacer delirar, excitar la agresividad o derrumbar los escrúpulos» ${ }^{(11)}$.

El poder cada vez mayor, por el monto de conocimientos que recibe el médico, lo pone en serio riesgo de inconductas profesionales. El médico es el sujeto moral de la medicina. En último análisis, dependerá de su conocimiento y controles morales si utiliza tal poder para hacer el bien o el mal a la sociedad. En tal óptica, el problema central de la ética en salud en pleno siglo XXI, se ubica en la persona del médico: ¿Quién es? ¿Cuál es su calibre ético? y ¿Cuán efectivos son sus controles morales en su ejercicio profesional? En suma, cómo se enfrenta a los riesgos que lo asedian en esa compleja entramada denominada mercado de salud.

Bajo la perspectiva expuesta, resultaría un esfuerzo inútil enseñar ética con un programa o estrategia única en todas las universidades del país. La realidad social que circunda a cada facultad de medicina es distinta y la extracción social -con subculturas diferentes- de los alumnos, también.

Por ello, si lo que deseamos es que los alumnos, al momento de egresar de la universidad estén en condiciones de ejercer profesionalmente con clara conciencia de su elevada misión en bien de la sociedad, debemos discriminar a quiénes les será suficiente un aprendizaje adecuado a 
través de cursos oficiales y quiénes requerirán estrategias docentes agregadas como soporte al desarrollo moral de su personalidad. De este modo, la enseñanza de la ética profesional no puede ser uniforme, como si se tratara de un curso más. Ella debe estar centrada en la persona del alumno, previa evaluación de su estructura moral, salud mental y de su entorno familiar y social.

Sumado a ello, el médico y, en general los profesionales de la salud deben ser conscientes de la magnitud del problema y de las condiciones de la sociedad a la que sirven. La nuestra, por ejemplo, afectada por variables de subdesarrollo y pobreza, corrupción y violencia, ya señaladas, imprime también su particular sello negativo a su trabajo profesional. Bajo estas circunstancias, las instituciones de salud -pilares de la sociedad- han sido, unas más, otras menos, afectadas por tales condiciones. Y ponen a prueba la responsabilidad de sus profesionales para defender, con su comportamiento ético, tanto a la organización hospitalaria a la que pertenecen, cuanto a sí mismos, del efecto deletéreo de tales influencias.

Un profesor sanmarquino, reconocido por su ejemplar conducta y calidad didáctica, contó en una reunión docente que al finalizar una clase sobre ética profesional ofreció la palabra al alumnado para preguntas y comentarios finales. Uno de ellos, con airada voz comentó «¿Cómo entender lo que usted nos ha explicado sobre los valores de la medicina cuando en el hospital donde hacemos prácticas vemos médicos que hacen todo lo contrario?»

Efectivamente, cómo superar el difícil trance de «enseñar» ética en una escuela médica y confrontar al alumno con una diferente "ética» en algunas instituciones de salud. Por ejemplo, recientemente los medios de comunicación han denunciado a dos hospitales, en el primero por una videograbación de colegas que en horas hospitalarias de atención se ausentaban del hospital para atender pacientes en una clínica privada vecina; y en el segundo, por denuncias formuladas sobre negociado de medicamentos entre médicos y pacientes. Tal inconveniente, en realidad depende de lo que denominamos el "Sistema Formador», conformado sistémicamente: «El enfoque sistémico permite comprender que en toda conducta o reacción humana la causalidad no es lineal ni obedece a un único factor sino que, por el contrario, es determinada por una variedad de factores provenientes de los diversos niveles del sistema en los cuales el sujeto existe. En nuestro concepto de sistema formador, incluimos como niveles a la sociedad, la universidad, la facultad de medicina, las sedes docentes hospitalarias, el cuerpo docente, el cuerpo administrativo y los alumnos. Todos ellos ejercen diversos grados de influencia en el discente como ser humano individual» (12).

\section{¿CUÁL ES LA MEJOR MANERA DE ENSEÑAR ÉTICA A LOS PROFESIONALES DE SALUD?}

A diferencia de antaño, cuando Don Gregorio Marañón afirmaba que no se requería, porque todo aquel que estudiara medicina por vocación era un individuo de textura moral intachable, hoy se acepta que tal empeño resulta fundamental, particularmente en el campo de las ciencias de la salud, en las que el profesional debe tomar decisiones no sólo sobre el bienestar sino sobre la vida del paciente, en una relación en la que ambos deliberan conjuntamente sobre la mejor opción a la luz de los valores morales en juego.

En el Perú, Cárdenas se ha ocupado del tema estudiando 22 escuelas de medicina peruanas. Todas ellas asignan al curso de ética, como asignatura independiente, 17 semanas (un semestre académico); 68 horas promedio para su dictado. Sólo tres escuelas cuentan con dos cursos, duplicando el tiempo asignado a la formación ética. Del total de créditos sólo les corresponde el 1,0\% (en las escuelas con un curso) y el $2,0 \%$ en las escuelas con dos. Concluye expresando que, «Hoy en día, la educación formal en ética médica es parte del plan de estudios de prácticamente todas las escuelas médicas del mundo, sin embargo, no existe un modelo único ni consenso sobre la mejor manera de educar en el tema» ${ }^{(13)}$.

Mendoza fundamenta la necesidad de incorporar la enseñanza de la ética médica y de la bioética clínica en los currículos de las facultades o escuelas de medicina, y discute los alcances de este proceso de enseñanza-aprendizaje, el momento de la formación en el que debe situarse, sus contenidos y métodos, y el rol del docente en el cumplimiento de sus objetivos educacionales y en la investigación (14).

Fernando Sánchez Torres, por su lado, afirma que la medicina es, de suyo, una actividad antropocéntrica y que la ética humanista, para saber lo que es bueno para el hombre, debe necesariamente conocer primero la naturaleza de éste. Advierte su preocupación sobre el hecho de que el médico se aleje, cada vez más, del hombre enfermo. Ya no lo toca para sentir su fiebre, no lo explora para recoger su angustia. No lo escucha en el acto médico y evita el coloquio porque no dispone de tiempo para hacerlo, olvidando que la palabra y el diálogo son los mejores instrumentos diagnósticos y terapéuticos (irremplazables por medios tecnológicos). «La culpa hay que endilgársela a esa tendencia mecanicista de la medicina actual, propiciadora de que todo lo hagan los aparatos» ${ }^{(15)}$

Y no olvidemos que en los centros de atención, no sólo los médicos y las enfermeras son responsables de la acción sobre el paciente. En ello participa todo el personal de la institución -profesional y administrativo- que si no 
está comprometida directamente con la ética médica sí lo está con principios éticos de carácter universal. Como son las relaciones interprofesionales e interpersonales que al infinito se suceden en la trama laboral hospitalaria creando y recreando su ethos institucional y su clima organizacional.

\section{ENSEÑANZA DE LA ÉTICA}

Toda ética como sistema dice Abel Naranjo Villegas en sus Disertaciones sobre Ética, está compuesta de unas reglas tendientes a conducir al hombre a su fin (a su logos). Pero así como orienta al paciente a su finalidad última, también lo hace con el médico, como profesional y persona. Ambos tienen derechos y deberes en el cumplimiento del acto médico, y corresponde al profesional, instruir al paciente en su respectivo rol para que colabore en beneficio de su salud. La ética profesional no es sólo médica, incluye a muchos profesionales de otras áreas.

Por ello, la responsabilidad social de toda facultad de medicina en el siglo $X X I$, no puede perder de vista que a la enseñanza médica se le está dando un enfoque predominantemente técnico, con claro descuido y, en ciertas áreas, prescindencia de lo humanístico, entendiendo por este término todo aquello que tiene que ver con el espíritu del hombre. Y la ética se vincula a la base espiritual del hombre.

El respeto por la vida y los fueros de la persona humana constituyen su esencia espiritual. Por consiguiente, el ejercicio de la medicina tiene implicaciones humanísticas que le son inherentes ${ }^{(16)}$. Ortiz ${ }^{(17)}$ ha señalado las siguientes connotaciones en el concepto de Ética: Como filosofía, en la cual se orienta a la discusión sobre la naturaleza de los principios y las normas morales; Como ciencia social, enderezándose a la explicación de los procesos históricos de moralización de la sociedad; y Como tecnología social, guiándose a la realización efectiva de los valores superiores en la sociedad y las personas.

No obstante, para insertar todo ello en el proceso educativo que nos preocupa resulta esencial precisar qué entendemos por el concepto «hombre», suscitando en el estudiante una intensa reflexión sobre lo distintivo del ser humano, su capacidad de comunicar mediante la palabra, el logos, su aptitud para la innovación y su naturaleza moral.

\section{HABRÍA DOS GRUPOS DE ALUMNOS CON DIFERENTES NECESIDADES DE ENSEÑANZA ÉTICA}

Estudios realizados en la Facultad de Medicina de la Universidad Nacional Mayor de San Marcos (UNMSM), institución que comprende cinco escuelas del área de la salud: Medicina, Enfermería, Obstetricia, Nutrición y Tecnología Médica (con aproximadamente 1500 estudiantes de pregrado en total) evidencian preocupantes prevalencias de vida de problemas de salud mental ${ }^{(18)}$. Es decir, que un cierto porcentaje del «sujeto de la enseñanza», el alumno de medicina y de otras ciencias de la salud, muestra indicadores negativos de salud mental y desarrollo moral, con desventajas personales en su contra.

Entre las patologías detectadas preocupan, particularmente, las prevalencias de vida de conducta suicida $(8,4 \%)$ e indicadores de conducta disocial (más altas que en la población general) y problemas con el alcohol, además de depresión y ansiedad ${ }^{(18)}$. Los hallazgos son concordantes con estudios previos realizados en la misma universidad ${ }^{(19,20)}$. Cabe precisar que, la conducta suicida, constituye actualmente un problema de salud pública a nivel mundial y ha producido más muertes que las dos guerras mundiales ${ }^{(21)}$.

Las conductas disociales, como su nombre lo indica, son comportamientos que van contra las normas sociales de convivencia e implican riesgo de un desarrollo moral negativo en la vida futura del sujeto. Aunque el instrumento utilizado en las encuestas sólo detecta «indicadores de conducta disocial», permitiéndonos hablar, en estos casos, de «probabilidad de» y no de «diagnóstico de», mal haríamos en negar que este grupo de alumnos adolescentes enfrentan mayor riesgo que otros de consolidarse en personas con un trastorno de personalidad disocial pasados los 18 años, de no modificar los elementos que intervienen en su dinámica ${ }^{(22)}$.

Frente a esta realidad, la reflexión que como profesores responsables debiéramos hacernos es la siguiente: ¿Los estudiantes así detectados serán, al concluir sus estudios, profesionales de bien para la sociedad? Durante los ocho años o más de estudios que estarán en la Escuela de Medicina, y seis los de las otras escuelas, ¿no podríamos hacer algo más por ellos?

Estos jóvenes no sólo necesitan recibir enseñanza curricular sobre problemas éticos, requieren fundamentalmente, estrategias educativas que aspiren a proveerles soporte al desarrollo moral de su personalidad.

\section{NADIE PUEDE DAR LO QUE NO TIENE: EL SISTEMA FORMADOR COMO MODELO DEL ESTUDIANTE}

Para tratar como persona y formar a sus alumnos como personas de comportamiento ético debemos nosotros los profesores, primero, aprender a ser personas y mostrar con nuestra conducta ética la coherencia moral básica que existe entre nuestras acciones y nuestros pensamientos.

Nuestra tesis fundamental es que la formación ética y humanista del discente dependerá del impacto que sobre él/ella produzca el sistema formador, es decir, la ética institucional donde se ha formado (o deformado) a lo largo 
de siete o más años de su existencia, parte de la cual transcurre durante su ciclo vital de adolescente.

\section{REFLEXIONES FINALES}

La preocupante realidad descrita obliga, moral y científicamente a investigadores y docentes, a ensayar acciones de solución. Una posibilidad, intentada por nuestro grupo, se apoyó en la asociación positiva entre intento suicida y «conflictos con los padres». Previos estudios habían destacado el fracaso de la figura paterna en la dinámica familiar. En 1985, por ejemplo, un estudio sobre la relación madre-hijo en jóvenes de ambos sexos, de 10 a 15 años de población urbano-marginal, se encontró una figura paterna muy depreciada, el $58 \%$ de los hijos varones informaba «no querer ser como su padre») (23).

Tal sustento teórico llevó al grupo de investigadores del Instituto de Ética en Salud de la Facultad de Medicina de la Universidad Nacional Mayor de San Marcos, a elaborar un programa tutelar especializado para alumnos con dificultades de salud mental. Con tal propósito se entrenó a un grupo de profesores, para ofrecerles, en forma individual, orientación académica y personal, además de apoyo emocional continuado durante la carrera y oportunidad de diálogo sobre problemas particulares. El programa ofreció éxitos individuales pero el seguimiento resultó complicado. Aspectos difíciles de manejar fueron: las recargadas tareas académicas, la lejanía de las viviendas de los alumnos que consumían muchas horas en su transporte dejando poco espacio libre para otras actividades y dificultades de coordinación horaria entre profesores y alumnos para sostener entrevistas regulares. El programa se detuvo y sobre la experiencia ganada, se intenta, ahora, reiniciarlo con apoyo tecnológico.

\section{CONCLUSIÓN}

Consideramos que la enseñanza de la ética debe planificarse para dos grupos de alumnos con diferentes necesidades: aquellos que sin patología de salud mental pueden beneficiarse con la estrategia curricular regular; y aquellos que, presentando problemas de salud mental e indicadores de dificultades en su conducta social, deben, además, ser ayudados con técnicas orientadas a ofrecerles apoyo en su desarrollo moral. El reto de incluir una enseñanza ética con un componente general y un componente individualizado centrado en las necesidades morales del estudiante como persona debe ser asumido por la universidad del siglo XXI y las intervenciones resultantes evaluadas para generar evidencia que contribuya a la mejora y posible replanteo del modelo de educación en ética para la formación del profesional de salud bajo la pregunta de ¿Qué necesita nuestro país, profesionales que sepan ética o que se comporten éticamente?.

Fuentes de financiamiento: autofinanciado.

Declaración de conflictos de interés: el autor no refiere conflictos de interés.

\section{REFERENCIAS BIBLIOGRÁFICAS}

1. World Federation for Medical Education. Estándares Globales en Educación Médica. Educ Méd. 2004;7(Supl 2):5-6.

2. Quintana Villlar C. Algunos aspectos de la enseñanza de la Bioética. Boletín de la Escuela de Medicina, Pontificia Universidad Católica de Chile. 1998;27:24-26.

3. Gracia D. The mission of ethics teaching for the future. International Journal of Ethics Education. 2016;1(1):7-13. doi: https://doi.org/10.1007/s40889-0150008-1.

4. Ortiz P. La Formación de la Personalidad. Lima: Dimas Editores; 1996.

5. McDonald GM, Donleavy GD. Objections to the teaching of business ethics. J Bus Ethics. 1995;14:839-853. doi: https://doi.org/10.1007/BF00872350.

6. Fatone V. Introducción al existencialismo Segunda edición. Columba. Buenos Aires. 1953.

7. Perales A. Salud Mental y Desarrollo. Anales de Salud Mental, 1988;IV(12):125-134.
8. Perales A. Concepto de Salud Mental: La experiencia peruana. Anales de Salud Mental. 1989;V(1-2):103-110.

9. Perales A. Salud Mental: Variables Socioeconómicas, Políticas y Culturales. Anales de Salud Mental. 1993(1-2):83-107.

10. Ortiz P. Ética Social. Para el desarrollo moral de las instituciones educacionales y de salud. Universidad Nacional Mayor de San Marcos, Lima, 2007.

11. Betto F. La Mosca Azul. Ocean Press, Australia, 2011.

12. Perales A. Ética y Humanismo en la formación médica. Acta Bioethica. 2008; XIV(1):30-38.

13. Cárdenas M. La educación ética en las escuelas de medicina peruanas: un estudio de sílabus. Tesis de Magíster. Universidad Nacional Mayor de San Marcos. 2012. Lima. Perú.

14. Mendoza A. Fundamentación de la enseñanza de la ética médica y la bioética clínica. Acta méd peruana. 2009;26(2):131-133.

15. Sánchez Torres F. La ética humanista, Capítulo VI: Ética médica y Humanización en salud [Internet]. [citado el 2 de febrero de 2019]. Disponible en: https://encolombia.com/libreria-digital/lmedicina/ letica-medica/etica-medica-capitulo-iv/

16. Colombia, Congreso Nacional. Ley 23 del 18 de febrero de 1981 [Internet]. [citado el 2 de febrero de 2019]. Disponible en: https://www.mineducacion.gov.co/1621/ articles-103905_archivo_pdf.pdf.

17. Ortiz P. Educación y formación de la personalidad Fondo Editorial UCH. Lima, 2008

18. Perales A, Sánchez E, Barahona L, Oliveros M, Bravo E, Mendoza A, et al. Conducta y personalidad disociales en estudiantes de medicina: implicancias para la educación médica peruana. An Fac med. 2018;79(3):238-43. doi: http:// dx.doi.org/10.15381/anales.v79i3.15018

19. Perales A, Acevedo L, Gutiérrez W, Sánchez E, Oblitas B, León $\mathrm{P}$, et al. Conducta suicida en estudiantes de las escuelas de educación y educación física de la Universidad Nacional Mayor de San Marcos. Investigación Educativa. 2013;17(2):185-194. 
20. Perales A, Sánchez E, Parhuana A, Carrera R, Torres H. Conducta suicida en estudiantes de la escuela de nutrición de una universidad pública peruana. Rev Neuropsiquiatr. 2013;76(4):231-235

21. Organización Mundial de la Salud. Informe mundial sobre la violencia y la salud [Internet]. Geneva: OMS; 2002 [citadoel2defebrerode2019].Disponible en: https://apps.who.int/iris/bitstream/ handle/10665/112670/9275315884 spa.pdf ?sequence $=1$.

22. Organización Mundial de la Salud. CIE-10 Trastornos mentales y del comportamiento: Descripciones clínicas y pautas para el diagnóstico. Madrid: Meditor; 1992.

23. Perales A, Tejada K, Villanueva M,HayashiS. Relación madre-hijo en una población urbano-marginal. Estudio Piloto. Anales de salud mental. 1985;1(1-2):50-68.

Correspondencia: Alberto Perales Cabrera Correo electrónico:perales.alberto@gmail.com Av. Javier Prado Oeste 445 - Dpto 101, San Isidro, Lima, Perú

Teléfono 441-2239

\section{¿CÓMO ENVIAR UN ARTÍCULO A NUESTRA REVISTA?}

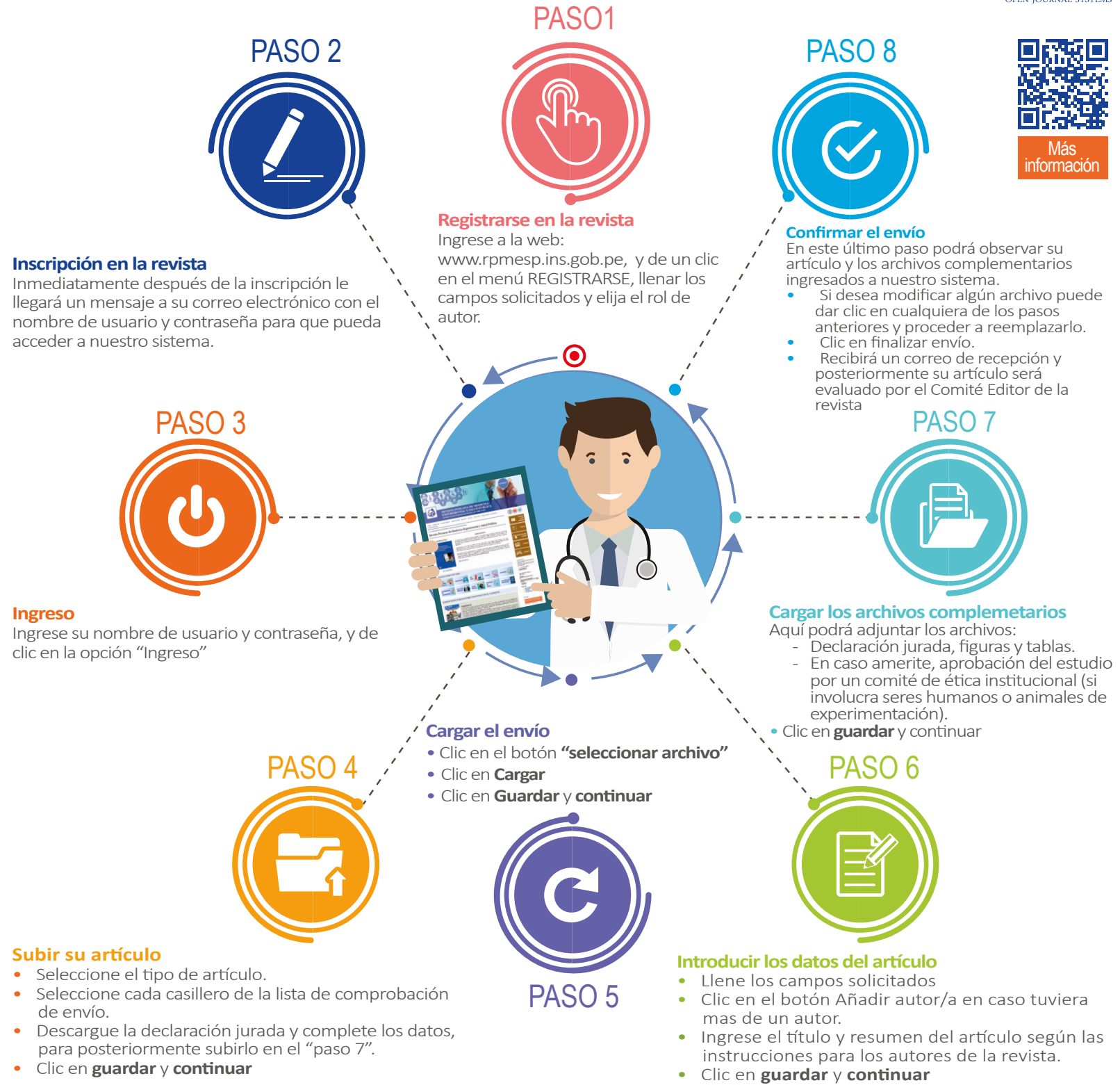

